\title{
Les chasses franco-suisses sur le Haut-Rhône Réflexions sur les opérations de 1987
}

\author{
C. Ricard et L. Levasseur
}

Compagnie nationale du Rhône

\section{Objet des opérations en Suisse et en France}

Les premiers ouvrages hydro-électriques situés sur le Rhône suisse en aval du confluent de l'Arve sont les barrages de Verbois et Chancy-Pougny.

La part des matériaux en suspension apportés par l'Arve qui se décante dans leurs retenues est estimée à 400 tonnes par an en moyenne. Si ces dépôts n'étaient pas évacués régulièrement, le remous de l'Arve en crue s'élèverait au quartier de la Jonction Genève et aggraverait les inondations [1].

Cette évacuation est réalisée au moyen de vidanges périodiques de la retenue de Verbois. Les Services industriels de Genève procèdent à ces vidanges depuis 1945 (soit deux ans après la mise en service de Verbois). La périodicité actuelle de ces vidanges est de trois ans.

Parmi les matériaux remobilisés au cours de la vidange de la retenue de Verbois, ceux qui arrivent à la frontière sont essentiellement des matériaux fins dont la dimension granulométrique est pour plus de $90 \%$ d'entre eux inférieure à $200 \mu$, le diamètre médian (d50) variant au cours des chasses entre $15 \mu$ et $25 \mu$.

Pour les aménagements français, les opérations consistent à éviter le stockage massif de ces matériaux dans les retenues en recherchant de bonnes conditions de transport, et en assurant une diffusion des matériaux qui ne provoque pas de dommages à l'environnement. Ces manœuvres doivent rester compatibles avec les besoins des différents usagers du Rhône. Les chasses de 1987 ont concerné 1,5 million de tonnes de matériaux.

\section{Les manœuvres françaises et les consignes à respec- ter}

La chaîne des aménagements hydro-électriques du HautRhône comporte 6 aménagements. Celui de tête, Génissiat, est un barrage-usine de moyenne chute, $67 \mathrm{~m}$ environ en eaux moyennes. Les cinq autres aménagements sont des ouvrages de basse chute, les quatre plus aval comportent des dérivations usinières.

Pour réduire les dépôts, les niveaux des retenues sont abaissés en vue d'obtenir des vitesses plus favorables au transit.

Grâce aux organes de vidange de Génissiat, il est possible d'assurer un dosage moyen du taux de matières en suspension (MES) à l'aval de cet ouvrage en répartissant judicieusement les débits entre la vanne de fond et la vanne de demi-fond situées respectivement en rive droite et en rive gauche de la retenue à des cotes différentes $(23 \mathrm{~m}$ d'écart). Ces vannes évacuent, de ce,fait, des eaux dont les taux de MES sont très différents.

Le contrôle des concentrations permet les ajustements nécessaires pour respecter la consigne qui impose les seuils ci-dessous au pont de Seyssel, situé à une vingtaine de km en aval de Génissiat. La concentration en MES n'y doit jamais être supérieure à $10 \mathrm{~g} / \mathrm{l}$ pendant plus de $6 \mathrm{~h}$ consécutives, et $15 \mathrm{~g} / 1$ pendant plus de $30 \mathrm{~min}$ consécutives. La moyenne à respecter au cours des chasses est de $5 \mathrm{~g} / \mathrm{l}$.

En outre, pendant les chasses sur les quatre aménagements comportant des dérivations usinières, les eaux

\section{Upper Rhône flushes - Remarks on the activities of 1987}

Flushes carried out once every three years on the Swiss part of the Rhone river are aimed at the removal of sediment deposits brought by the Arve river into the Verbois reservoir. On the French upper Rhone, the transit of the resulting suspended load is to be secured in the best conditions possible as regards their effect on the natural environment and riparian inhabitants. The Compagnie Nationale du Rhone monitors the sediment flows from the outlets of the Genissiat dam which is the first French scheme downstream from the border. Flushes of june 1987 were carried out in periods of significant flows. They made up an interesting experience for the knowledge of the turbidity current through the Genissiat dam bottom outlet. They also permitted to verify the effectiveness of the operation rules set up in accordance with the instructions of the Rhone-Alpes Authorities Technical water committee. 
doivent transiter prioritairement par elles en évitant les déversements aux barrages. (Il s'agit d'une obligation réglementaire pour Belley et Brégnier-Cordon et d'un objectif pour Chautagne et Sault-Brénaz.)

Cette contrainte conduit à assurer un débit entrant dans chacun de ces quatre aménagements au plus égal au débit turbinable soit $700 \mathrm{~m}^{3} / \mathrm{s}$, ce qui nécessite évidemment des conditions hydrologiques favorables.

\section{Les utilisations des vannes de fond et $1 / 2$ fond au cours des chasses successives}

Jusqu'en 1980, date de la mise en eau de la retenue de Chautagne, Génissiat et Seyssel étaient les seuls ouvrages sur le Haut-Rhône français. De 1954 à 1978 la vanne de fond de Génissiat a pu être manœuvrée au cours des chasses, mais elle ne participait pas à la chasse. Ces manœuvres étaient destinées à vérifier son fonctionnement et à éviter notamment son éventuel blocage par les matériaux déposés dans la galerie de fond.

Ces manœuvres avaient pour effet de provoquer momentanément des pointes du taux de matières en suspension en aval de Génissiat ce qui, pensait-on, interdisait d'ouvrir la vanne de fond de façon continue. C'est ainsi que la majeure partie des matériaux sortant de Génissiat était évacuée par la vanne de demi-fond (les turbines étant toujours à l'arrêt pendant ces opérations).

La manœuvre de Génissiat consistait donc à abaisser la retenue, avant l'arrivée des matériaux provenant de Verbois et Chancy-Pougny, jusqu'à un niveau qui était conservé ensuite durant tout le passage du flux chargé par de la vanne de $1 / 2$ fond.

L'expérience des chasses successives avait déterminé la cote du plan d'eau permettant un compromis entre le volume de matériaux évacués à l'aval et les nuisances produites. Il fallait admettre, en contre-partie, un dépôt important de l'ordre de $50 \%$ dans la retenue. De plus ce processus ne permettait aucunement d'intervenir, en cours d'opération, sur la concentration des MES qui transitaient vers l'aval.

En 1984, la Compagnie Nationale du Rhône a proposé d'ouvrir et de manœuvrer la vanne de fond de Génissiat dès le début des chasses pour respecter les seuils de concentration imposés au pont de Seyssel et rappelés ci-avant au paragraphe II.

Cette proposition découlait des observations faites au cours des chasses précédentes au moment de l'ouverture de la vidange de fond. Il avait été constaté en effet que si cette ouverture entrainait bien un pic de MES à l'aval la concentration diminuait rapidement ensuite. Ces ouvertures étaient réalisées alors que des matériaux venaient de décanter à proximité de la vidange de fond.

On pouvait donc supposer que si l'on ouvrait partiellement cette vidange de fond assez tôt, pendant la phase d'abaissement du plan d'eau, le flux de matériaux allait principalement transiter par elle. Le taux de MES obtenu en cours de chasse, y serait sans doute élevé mais il serait possible de le réduire à l'aval, avec de l'eau moins chargée, déversée par la vidange de $1 / 2$ fond.

Cela exigeait de connaître les taux de MES sortant par les deux vannes fond et demi-fond, d'effectuer la modulation des débits et de vérifier en temps réel à l'aval que la distribution calculée satisfaisait aux consignes imposées au pont de Seyssel.

Les manœuvres réalisées en 1984 ont montré, en effet, que l'ouverture continue de la vidange de fond crée, avec un abaissement approprié de la retenue, un courant de densité qui transite par cette vidange et que le contrôle du taux de MES aval est réalisable par la modulation des débits. Pour amorcer ce courant il faut cependant abaisser la retenue d'une dizaine de mètres environ en dessous de sa cote d'exploitation minimale soit au voisinage de la cote $315 \mathrm{NGF}$.

Les chasses de 1984, par ce procédé de "conduite des manœuvres en fonction des MEST constatées en aval de Génissiat " ont atteint exactement les objectifs prescrits à la suite des accords intervenus avant les chasses, avec les autorités et les riverains [3].

\section{Les observations faites à l'occasion des manœuvres de 1987}

Les vidanges de Verbois et de Chancy-Pougny, prévues du 13 au 16 juin 1987, ont été réalisées au cours d'une période de forte hydraulicité du Rhône, le règlement international qui prévoit ces opérations imposant un calendrier contraignant. Les opérations commencées normalement ont été perturbées par les crues.

Les forts débits du Rhône, de l'Arve et du Fier ont ainsi conduit à utiliser la retenue de Génissiat comme réservoir écréteur pour ne pas dépasser $700 \mathrm{~m}^{3} / \mathrm{s}$ et éviter les déversements aux barrages situés en aval de Seyssel. Le stockage de l'eau, et donc la remontée du plan d'eau de Génissiat, ont débuté dès le dimanche 14 juin alors que les premiers matériaux issus des chasses de Verbois arrivaient à peine à Pougny où se trouve la station de mesure des MES franchissant la frontière (graphe 1).

Lorsque cette remontée du plan d'eau a débuté le courant de densité passant par la vidange de fond était bien établi et était alimenté par les matériaux remobilisés en bout de retenue.

A $6 \mathrm{~h} 30$ le 14 juin il y avait $4,8 \mathrm{~g} / \mathrm{l}$ par la vidange de fond et $2,5 \mathrm{~g} / 1$ par celle de $1 / 2$ fond ((graphe 1). Cette remontée quoiqu'irrégulière n'a eu aucun effet sur le courant de densité qui s'est maintenu jusqu'à la fermeture de la vanne de fond, le 15 juin à $15 \mathrm{~h}$, soit pendant une quarantaine d'heures.

Le graphe 1 montre en effet que le courant de fond était toujours établi le 15 juin à $13 \mathrm{~h}$, alors que la retenue était au-dessus de la cote $320 \mathrm{NGF}$ et qu'il a retransmis, environ $7 \mathrm{~h}$ après et assez fidèlement, la pointe de MES observée à Pougny le 15 juin à $6 \mathrm{~h}$. 

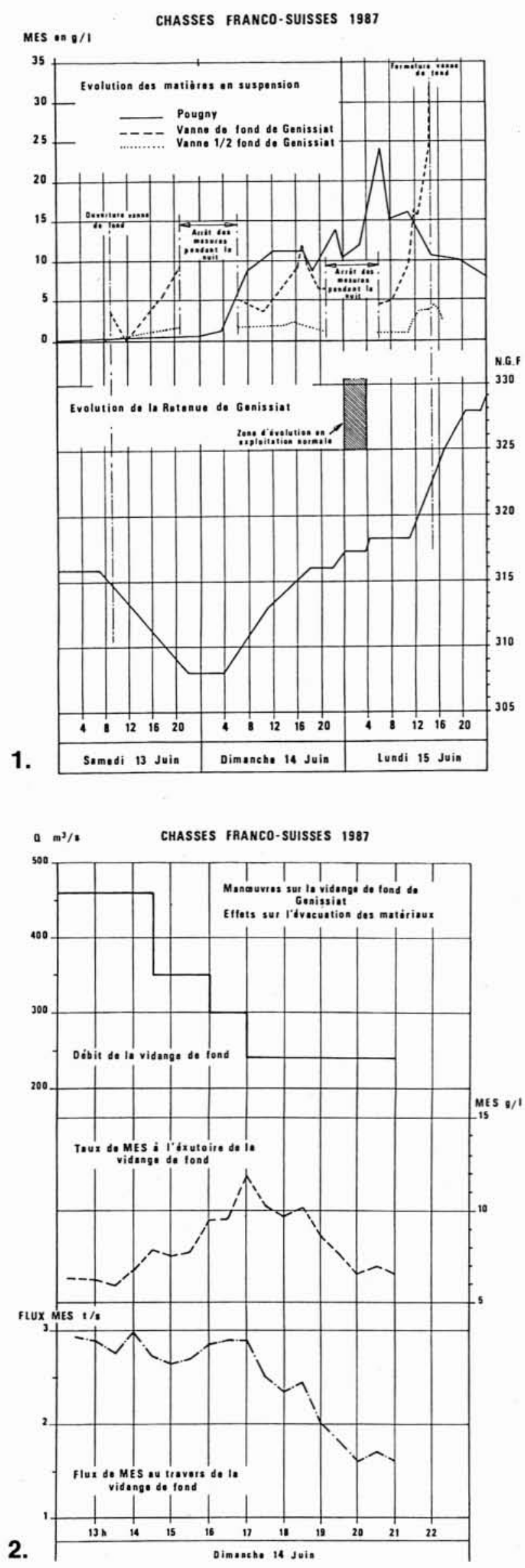

On peut conclure que le courant de densité au fond, une fois établi, persiste indépendamment des mouvements de la retenue même si celle-ci montre au-dessus du niveau d'amorçage de ce courant.

Cette constatation ouvre de nouvelles perspectives pour la conduite des chasses : Les ouvrages de Génissiat permettent désormais en effet non seulement de contrôler le taux de MES à l'aval, mais aussi, dans une certaine mesure, les débits sans perte du contrôle du taux de MES grâce à des variations de niveau de la retenue. Cette constatation pourra être notamment mise à profit pour commencer le remplissage de la retenue de Génissiat, pendant la phase de vidange des retenues de Verbois et Chancy-Pougny, en année de faible hydraulicité.

La chasse de juin 1987 a conduit à faire une autre observation notable : la nécessité de réguler le débit en aval de Génissiat en fonction des apports du Rhône et des versants a conduit à effectuer de nombreuses manœuvres sur la vidange de fond. Celles-ci ont permis de mettre en évidence que la manœuvre de réduction de débit est accompagnée de phénomènes transitoires sur les taux de la concentration en MES de la veine liquide qui débouche en aval. C'est sur les manœuvres du 14 juin après-midi que ce phénomène est le plus caractéristique, comme le montre le graphe $n^{\circ} 2$.

De 14 h 30 à 17 h le débit de la vidange de fond a été réduit, par paliers, de $460 \mathrm{~m}^{3} / \mathrm{s}$ à $240 \mathrm{~m}^{3} / \mathrm{s}$. Parallèlement la concentration de la veine augmentait tandis que le flux de matériaux en suspension évacué par la vanne de fond restait pratiquement constant pendant ces deux heures et demie. La concentration en MES de la veine liquide retrouve les valeurs initiales de 6 à $7 \mathrm{~g} / 1,2$ à $3 \mathrm{~h}$ après la dernière manœuvre de réduction de débit.

Il existerait donc une sorte d'inertie du flux de MES au travers de la vidange de fond, qui provoquerait, simultanément à la réduction du débit, une augmentation transitoire du taux de MES dans la veine sortant en aval.

Ce phénomène pourrait expliquer à la sortie de la vanne de fond la forte variation jusqu'à $32 \mathrm{~g} / \mathrm{l}$, obtenue le 15 juin juste avant sa fermeture alors que la pointe mesurée à Pougny avec précision $7 \mathrm{~h}$ auparavant était de $26,6 \mathrm{~g} / \mathrm{l}$. En effet la fermeture totale de la vanne a été précédée de fermetures partielles entre $12 \mathrm{~h} 30$ et $13 \mathrm{~h} 45$ pour diminuer le débit de $460 \mathrm{~m}^{3} / \mathrm{s}$ à $100 \mathrm{~m}^{3} / \mathrm{s}$. Ces manœuvres ont donc pu avoir comme effet ce phénomène transitoire de reconcentration en MES de la veine.

\section{Mesures effectuées sur les matériaux en suspension}

Des prélèvements ont été réalisés en différents points caractéristiques du Rhône pour mesurer la granulométrie des sédiments transportés.

Pougny est la station de référence à la frontière. Elle permet de caractériser les sédiments entrant dans la retenue de Génissiat. Pyrimont est la première station de mesure après le barrage de Génissiat. 
La quantité d'échantillons prélevés et les événements survenus pendant les opérations de 1987 permettent de distinguer les cas suivants :

- caractéristiques des sédiments à la frontière en fortes eaux, avant l'apport des matériaux provenant des chasses de Verbois et Chancy-Pougny;

- caractéristiques des sédiments provenant des chasses de ces aménagements ;

- caractéristiques des sédiments par fortes eaux en aval de Génissiat en dehors de la période des chasses ;

- caractéristiques des sédiments remis en suspension dans la retenue de Génissiat par l'ouverture de la vidange de fond et l'abaissement de la retenue;

- caractéristiques des sédiments transportés par le courant de fond et provenant des chasses de Verbois;

- caractéristiques des sédiments provenant des chasses de Verbois qui transitent, en crue, par les groupes en exploitation à niveau normal de la retenue.

Il apparaît donc que l'aménagement de Génissiat retient tous les matériaux de dimension supérieure à $50 \mu$ provenant des chasses de Verbois. Ce qui signifie que 15 à $20 \%$ au moins de matériaux en suspension passant à Pougny restent dans la retenue de Génissiat à chacune des chasses triennales.

Les matériaux qui transitaient en aval de Génissiat le 16 juin au matin alors que l'exploitation était redevenue classique, ont les mêmes caractéristiques que ceux qui passaient par la vanne de fond en cours de chasse.

L'utilisation actuelle de la vidange de fond ne permet donc pas d'évacuer une part significative des plus gros matériaux qui atteignent la retenue pendant la vidange de Verbois.

La concentration en MES est mesurée à l'aide d'un appareil baptisé "psychomètre CNR ". Son principe est basé sur la pesée d'un volume connu d'un échantillon d'eau et de sédiments contenus dans un flacon en verre de 11 appelé pycnomètre [2].

Une fois l'échantillon parvenu en laboratoire la mesure peut être faite en 30 secondes par un personnel sans formation particulière. Cette méthode est à la fois simple et rapide ce qui est un gage d'efficacité pour suivre pas à pas l'évolution des chasses et intervenir sur les organes de vidange de Génissiat.

\section{Les enseignements pratiques des opérations de 1987}

Les constatations faites en 1987 et exposées dans cette communication seront mises à profit pour la gestion des futures chasses.

Le point le plus important est la possibilité de contrôler simultanément le débit et le taux de la concentration en
SEDIMENTS A POUGKY AVNTT CBASSES

\begin{tabular}{|c|c|c|c|c|c|c|}
\hline \multirow[t]{2}{*}{ STATION } & \multirow{2}{*}{$\begin{array}{l}\text { Moabre } \\
\text { d'echan- } \\
\text { tillon }\end{array}$} & \multirow{2}{*}{$\begin{array}{l}\text { Refus superieurs } 200 \mathrm{u} \\
\text { en de la masse de } \\
\text { l'echantillon }\end{array}$} & \multicolumn{3}{|c|}{ Gane $0-200 \mu$} & \multirow{2}{*}{$\begin{array}{l}\text { K. . .S.DES } \\
\text { ECHXH- } \\
\text { TILLOWS } \\
\text { en } g / 1\end{array}$} \\
\hline & & & d90 & d50 & d10 & \\
\hline POUGNY & 1 & 0. & $90 \mu$ & $22.7 \mu$ & $5,5 \mu$. & 0.46 \\
\hline
\end{tabular}

SEDIKENTS A POUGNY EN COURS DE CEASSSES

\begin{tabular}{|c|c|c|c|c|c|c|}
\hline \multirow[t]{2}{*}{ STATION } & \multirow{2}{*}{$\begin{array}{l}\text { Monbre } \\
\text { d'teban- } \\
\text { tillon }\end{array}$} & \multirow{2}{*}{$\begin{array}{l}\text { Refus superieurs d200u } \\
\text { en \& de la masse de } \\
1 \text { 'echadtillon }\end{array}$} & \multicolumn{3}{|c|}{ Ganae $0-200 \nu$} & \multirow{2}{*}{$\begin{array}{l}\text { M.E.S.DES } \\
\text { rCHWH- } \\
\text { TILWONS } \\
\text { en } g / 1\end{array}$} \\
\hline & & & d90 & d50 & d10 & \\
\hline POUGXY & 9 & $1,5:-8$. & $60-70 \mu$ & $15 \nu-22 \mu$ & $3 \nu-4,5 \nu$ & $\begin{array}{l}1,7 \\
26,6\end{array}$ \\
\hline
\end{tabular}

SEDIRENTS DA AVAL DE GENISSIAT PMR TORTES EAUX

\begin{tabular}{|c|c|c|c|c|c|c|}
\hline \multirow[t]{2}{*}{ STATION } & \multirow{2}{*}{$\begin{array}{l}\text { Moabre } \\
\text { d'teban- } \\
\text { tillon }\end{array}$} & \multirow{2}{*}{ 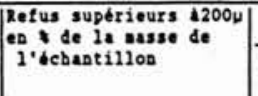 } & \multicolumn{3}{|c|}{ Gamse $0-200 \mu$} & \multirow{2}{*}{ 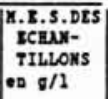 } \\
\hline & & & 890 & $d 50$ & d10 & \\
\hline PYRIMONT & 1 & $0,1 *$ & $56 \mu$ & $16,2 \mu$ & $3,5 \mu$ & 0,76 \\
\hline
\end{tabular}

SEDINENTS DE GEMISSIAT REMTS EM SUSPENSTON PAR U YTDNGE DE TOKD

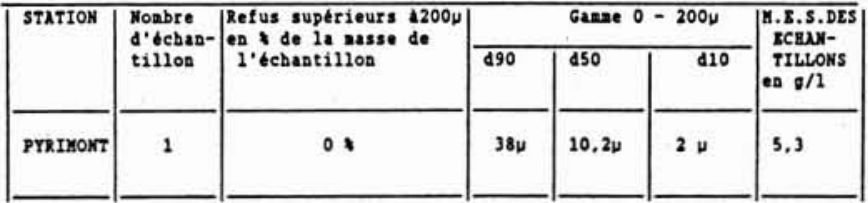

SEDTKENTS TRUSSPORTES PAR UE COURUYT DE TOND TE PROYTOAYCE DTS CEASSTS DE YERBOTS

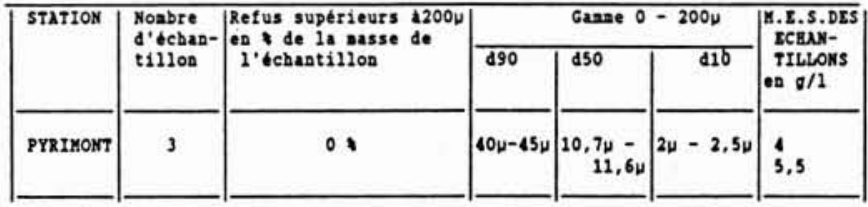

SEDTAENTS DN PROVENANCE DES CBASSES DE VERBOTS

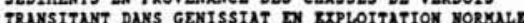

\begin{tabular}{|c|c|c|c|c|c|c|}
\hline \multirow[t]{2}{*}{ STATION } & \multirow{2}{*}{$\begin{array}{l}\text { Sonbre } \\
\text { d'bchan- } \\
\text { tillon }\end{array}$} & \multirow{2}{*}{$\begin{array}{l}\text { Refus superieurs d200W } \\
\text { en t de la masse de } \\
\text { l'echantillon }\end{array}$} & \multicolumn{3}{|c|}{ Gazae $0-200 \mathrm{H}$} & \multirow{2}{*}{$\begin{array}{l}\text { I.T.S.DES } \\
\text { ECENA- } \\
\text { TILLONS } \\
\text { ea } g / 1\end{array}$} \\
\hline & & & d90 & d50 & d10 & \\
\hline PYRIMONT & 1 & 0. & $45 \nu$ & $12,5 \mu$ & 2,64 & 1,36 \\
\hline
\end{tabular}

MES en aval de Génissiat. L'évacuation des matériaux à niveau constant ne s'impose plus.

L'inertie du flux de MES qui passe à travers la vanne de fond s'oppose aux manœuvres de celle-ci. Il est donc nécessaire d'anticiper les variations du flux de MES venant d'amont et donc de bien connaître l'évolution du taux de MES à Pougny et dans les vidanges de fond et $1 / 2$ fond. 


\section{Bibliographie}

[1] Rétention et évacuation des matériaux en transit au fil des retenues du Rhône aménagé. Par MM. Ricard et Barbet. Question IV, rapport 4, XIX Journées de l'hydraulique (Paris, septembre 1986).

[2] Détermination simple et rapide des MES par la méthode du pycnomètre CNR, application au contrôle des vidanges des retenues. MM. Niel, Collilieux, Giuliani. Revue française des sciences de l'eau, 4 (1985).
[3] Expérience acquise par EDF et CNR dans les vidanges des retenues, contrôles de la qualité des eaux. MM. Rambaud, Khalanski, Clair, Ricard. Comité français des grands barrages. Colloque technique. Paris, mars 1987 et Commission internationale des grands barrages, $16^{\circ}$ Congrès, San Francisco, 1988.

[4] "Transports et colères du Rhône" par MM. Cazenave et Pommier. Journées à Toulouse en hommage au Professeur Escande, décembre 1981.

\section{AMÉNAGEMENT DU HAUT-RHÔNE}

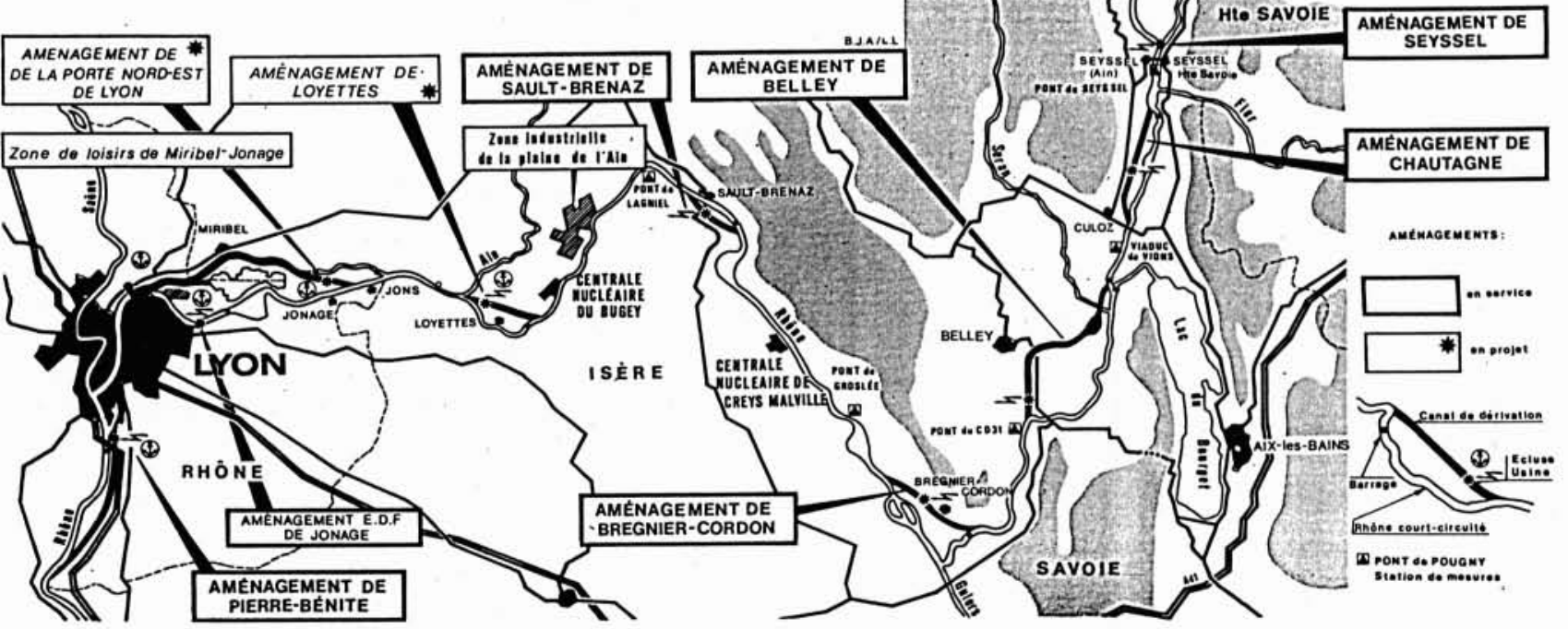

